\title{
Search for an opposite sign muon-tau pair and a $b$-jet at the LHC in the context of flavor anomalies
}

\author{
Debajyoti Choudhury, ${ }^{1, *}$ Nilanjana Kumar, ${ }^{1,2,3, \dagger}$ and Anirban Kundu ${ }^{4, \$}$ \\ ${ }^{1}$ Department of Physics and Astrophysics, University of Delhi, Delhi 110007, India \\ ${ }^{2}$ Saha Institute of Nuclear Physics, HBNI, I/AF Bidhan Nagar, Kolkata 700064, India \\ ${ }^{3}$ The Institute of Mathematical Sciences, HBNI, Taramani, Chennai 600113, India \\ ${ }^{4}$ Department of Physics, University of Calcutta, 92 A.P.C Road, Kolkata 700009, India
}

(Received 6 June 2019; published 1 October 2019)

\begin{abstract}
Extant anomalies in several semileptonic $B$-meson decays argue for physics beyond the Standard Model. Measurements of both neutral-current decays (such as $R_{K}, R_{K^{*}}$, and $B_{s} \rightarrow \phi \mu \mu$ ) as well as charged-current ones- $R(D)$ and $R\left(D^{*}\right)$ - provide strong hints for the violation of lepton flavor universality. Recent studies (D. Choudhury et al., Phys. Rev. Lett. 119, 151801 (2017), D. Choudhury et al., Nucl. Phys. B933, 433 (2018)) have shown that a class of effective field theory models may explain such anomalies in terms of only a few parameters which can be determined phenomenologically. In this literature, we examine such resolutions in the context of the requisite $(\bar{s} b)(\bar{\tau} \tau)$ operator and look for its signals at the $13 \mathrm{TeV} \mathrm{LHC}$, with a final state of one $b$-jet, and an oppositely charged $\mu$ - $\tau$ pair, with the muon coming from the decay of one of the $\tau$ leptons. We obtain discovery and exclusion limits on the model parameters as a function of the luminosity at the $13 \mathrm{TeV}$ LHC.
\end{abstract}

DOI: 10.1103/PhysRevD.100.075001

\section{INTRODUCTION}

Evidence from a multitude of experiments, such as $B A B A R$ [1], Belle [2-7], and, most recently, LHCb [8-13], suggests the presence of effects that violate lepton flavor universality, a cardinal principle within the Standard Model (SM). Related to several mesons containing the bottom (anti)quark, these "anomalies" appear in both charged- and neutral-current (NC) decays. For example, consider the ratios [14]

$$
R\left(D^{(*)}\right) \equiv \frac{\mathrm{BR}\left(B \rightarrow D^{(*)} \tau \nu\right)}{\mathrm{BR}\left(B \rightarrow D^{(*)} \ell \nu\right)}
$$

with $\ell=e$ or $\mu$, and, similarly,

$$
R_{J / \psi} \equiv \frac{\mathrm{BR}\left(B_{c} \rightarrow J / \psi \tau \nu\right)}{\mathrm{BR}\left(B_{c} \rightarrow J / \psi \mu \nu\right)}
$$

*debajyoti.choudhury@gmail.com

†nilanjana.kumar@gmail.com

*akphy@caluniv.ac.in

Published by the American Physical Society under the terms of the Creative Commons Attribution 4.0 International license. Further distribution of this work must maintain attribution to the author(s) and the published article's title, journal citation, and DOI. Funded by SCOAP.
While the SM estimates for the individual decays are already quite robust, the advantage of considering such ratios is that much of the remaining uncertainties, residing in the evaluation of the form factors, cancel out. Thus, any observed anomaly in such areas would be very intriguing, and we begin by recalling the experimental status.

The BABAR [1] measurements of $R(D)$ and $R\left(D^{*}\right)$, when taken together, exceed SM expectations by more than $3 \sigma$. Though the Belle measurements [2] lie a little below the $B A B A R$ measurements and are consistent with both the latter and the SM expectations, their result on $R\left(D^{*}\right)$ [3], with the $\tau$ decaying semileptonically, agrees with the SM expectations only at the $1.6 \sigma$ level. Similarly, the LHCb measurement [8] lies 2.1 $\sigma$ above the SM predictions. Taking into account all the measurements and their correlations, the disagreement between the data and SM is at nearly $3.8 \sigma[15,16]$.

The four-fermi effective interaction responsible for $B \rightarrow K^{(*)} \ell \nu$, namely, $b \rightarrow c \ell \nu$, is also responsible for driving $B_{c} \rightarrow J / \psi \ell \nu$ and analyzing $3 \mathrm{fb}^{-1}$ data. The $\mathrm{LHCb}$ Collaboration found

$$
R_{J / \psi}= \begin{cases}0.71 \pm 0.17 \pm 0.18 & (\exp ) \\ 0.283 \pm 0.048 & (\mathrm{SM})\end{cases}
$$

where the SM prediction [17-19] includes uncertainties accrued from the $B_{c} \rightarrow J / \psi$ form factors and is, thus, quite robust. While the level of the discrepancy is only at 
the $2 \sigma$ level, ${ }^{1}$ it is interesting to note that it points in the same direction as the others.

An opposite effect is seen for the neutral-current transitions, namely, $b \rightarrow s \ell^{+} \ell^{-}$. Once again, ratios of such decays constitute robust variables leading us to consider

$$
R_{K^{(*)}} \equiv \frac{\mathrm{BR}\left(B \rightarrow K^{(*)} \mu^{+} \mu^{-}\right)}{\operatorname{BR}\left(B \rightarrow K^{(*)} e^{+} e^{-}\right)} .
$$

While the SM predictions for both $R_{K}$ and $R_{K^{*}}^{\text {central }}$ are almost indistinguishable from unity [20-24], that for $R_{K^{*}}^{\text {low }}$ is $\sim 0.9$ (mainly due to the non-negligible $m_{\mu}$ ). The calculations are very precise with only minuscule uncertainties. The earlier result on $R_{K}[11,12]$ has recently been superseded by the LHCb Collaboration [13]:

$$
R_{K}=0.846_{-0.054-0.014}^{+0.060+0.016}, \quad q^{2} \in[1.1: 6] \mathrm{GeV}^{2} .
$$

For $R_{K^{*}}$, while the earlier LHCb data [11,12], viz.

$$
\begin{aligned}
R_{K^{*}}^{\text {low }} & =0.66_{-0.07}^{+0.11} \pm 0.03, & & q^{2} \in[0.045: 1.1] \mathrm{GeV}^{2}, \\
R_{K^{*}}^{\text {central }} & =0.69_{-0.07}^{+0.11} \pm 0.05, & & q^{2} \in[1.1: 6] \mathrm{GeV}^{2},
\end{aligned}
$$

were significantly away from the SM predictions, the recent Belle results [7], taking average over $K^{* 0}$ and $K^{*+}$ modes, are more compatible with the SM:

$$
R_{K^{*}}=0.94_{-0.14}^{+0.17} \pm 0.08, \quad q^{2} \in[0.045,] \mathrm{GeV}^{2} .
$$

While this can be construed as the $R_{K^{*}}$ average moving closer to the SM (note, though, the larger errors in the Belle results), an anomaly is still hinted at, with the magnitude of the deviation being somewhat less than that in the $R_{K}$ data.

A corroborating deviation is seen in $B_{s} \rightarrow \phi \mu \mu$ [25-27], namely,

$$
\begin{aligned}
& \left.\frac{d}{d q^{2}} \mathrm{BR}\left(B_{s} \rightarrow \phi \mu \mu\right)\right|_{q^{2} \in[1: 6] \mathrm{GeV}^{2}} \\
& \quad= \begin{cases}\left(2.58_{-0.31}^{+0.33} \pm 0.08 \pm 0.19\right) \times 10^{-8} \mathrm{GeV}^{-2} & (\exp .), \\
(4.81 \pm 0.56) \times 10^{-8} \mathrm{GeV}^{-2} & (\mathrm{SM}),\end{cases}
\end{aligned}
$$

where $q^{2}=m_{\mu \mu}^{2}$. This suggests that the discrepancies in $R_{K}$ and $R_{K^{*}}$ have been caused by a depletion of the $b \rightarrow s \mu^{+} \mu^{-}$ channel rather than an enhancement in $b \rightarrow s e^{+} e^{-}$. Such a conclusion is lent further weight by the long-standing $P_{5}^{\prime}$

\footnotetext{
${ }^{1}$ Given the smaller production cross section, the large uncertainty is understandable. This is expected to improve a lot once more data are analyzed.
}

anomaly [28] in the angular distribution of $B \rightarrow K^{*} \mu \mu$, with a more than $3 \sigma$ mismatch between the data and SM prediction. $^{2}$

Faced with all these anomalies, two approaches are possible. The first would be to construct an elaborate ultraviolet-complete theory. Examples are offered by $Z^{\prime}$ models [30] with flavor-violating couplings with the quarks and the leptons [31-36] on the one hand and, on the other, the exchange of leptoquarks [37-39] or, equivalently, sfermions in $R$-parity-violating supersymmetric models [40-42]. The alternative is to take recourse to an effective field theory (EFT) description, wherein only a set of Wilson coefficients are altered from their SM values [43-49]. In either case, one would, naively, expect that a sufficiently large set of unknown parameters (and/or fields) would need to invoked so as to enable the simultaneous explanation of all the anomalies while maintaining the rest of the well-tested SM phenomenology. However, if the nature of the UV theory (operative at a scale higher than the electroweak scale), the integrating out of whose heavy degrees of freedom is supposed to have given us the EFT, is entirely ignored, then a phenomenologically motivated EFT with only a small number of parameters needs to be considered. It has been shown [50-52] that such a minimal set of new physics (NP) operators, accompanied by a single lepton-mixing angle, can indeed explain almost all the observables adequately. More interestingly, the natural scale for such an explanation is seen to be a few TeVs, opening the interesting possibility of signatures at the LHC and/or future colliders.

In the present case, instead of attempting a generic study, we consider a particular signature, at the LHC, prompted by the scenarios discussed in Refs. [50,51]. Some such studies have been attempted in the past but in entirely different contexts, both at the simulation level [53-60], as well as by the ATLAS and CMS Collaborations, who have searched for flavor-violating signatures with dilepton final states [61-66]. We focus on a model which can explain, simultaneously, both the $\mathrm{CC}$ and the $\mathrm{NC}$ anomalies in semileptonic $B$ decays, for example, one with an enhanced $(\bar{s} b)(\bar{\tau} \tau)$ operator. We have studied the telltale signatures which include an opposite signed $\mu-\tau$ pair and a $b$-jet, induced by this operator when one tau decays to a muon. One of the novelties of this channel is that it does not suffer from a very large background unlike opposite sign same flavor lepton-pair signatures. Apart from effecting a full simulation, we also validate our background estimation with Ref. [66]. This, as well as our choice of a robust set of observables, renders our methodology applicable to a very wide class of NP scenarios.

The rest of the paper is constituted as follows. In the next section, we briefly discuss the EFT framework. In Sec. III, we study the collider signatures of the particular channel

\footnotetext{
${ }^{2}$ Recently, however, it has been argued in Ref. [29] that these discrepancies may have their origin, instead, in some new physics in $b \rightarrow s e^{+} e^{-}$.
} 
with detail analysis of the signal and background at the $13 \mathrm{TeV}$ LHC. Then, in Sec. IV, we discuss the discovery and exclusion perspectives of this particular channel. Last, we conclude by predicting some future possibilities in Sec. V.

\section{EFFECTIVE THEORY MODEL}

Considering all NP effects to be parametrized by $S U(3)_{c} \otimes S U(2)_{L} \otimes U(1)_{Y}$ invariant four-fermi operators, one needs at least two such structures [51] so as to both explain the anomalies and be consistent other lowenergy observables. While Refs. [50,51] did consider several possibilities, they identified certain combinations as favored scenarios. Subsequently, Ref. [52] reexamined the data, taking into account all correlations and, apart from establishing these scenarios (modulo certain alterations in the allowed parameter space), found that even the combinations dismissed in Ref. [50] can be accommodated. Rather than examine each such scenario, we consider a particular representative case, termed "model IV" in Ref. [50]. Analyses for the other scenarios can also be effected analogously. The Hamiltonian for the new physics can be expressed in terms of two operators involving lefthanded doublets $Q_{2 L}, Q_{3 L}$, and $L_{3 L}$ and right-handed singlet $\tau_{R}$ as

$$
\begin{aligned}
\mathcal{H}^{\mathrm{NP}}= & \sqrt{3} A_{1}\left[-\left(\bar{Q}_{2 L} \gamma^{\mu} Q_{3 L}\right)_{3}\left(\bar{L}_{3 L} \gamma^{\mu} L_{3 L}\right)_{3}\right. \\
& \left.+\frac{1}{2}\left(\bar{Q}_{2 L} \gamma^{\mu} L_{3 L}\right)_{3}\left(\bar{L}_{3 L} \gamma^{\mu} Q_{3 L}\right)_{3}\right] \\
& +\sqrt{2} A_{5}\left(\bar{Q}_{2 L} \gamma^{\mu} Q_{3 L}\right)_{1}\left(\bar{\tau}_{R} \gamma^{\mu} \tau_{R}\right)+\text { H.c. },
\end{aligned}
$$

where $A_{1,5}$ are unknown coefficients of mass dimension -2 and to be determined phenomenologically. For the sake of simplicity, we assume these to be real. The subscripts " 3 " and "1" represent the $S U(2)_{L}$ triplet and singlet currents, respectively. These can be expressed in terms of component fields as

$$
\begin{aligned}
\mathcal{H}^{\mathrm{NP}}= & \frac{3 A_{1}}{4}(c, b)\left(\tau, \nu_{\tau}\right)+\frac{3 A_{1}}{4}(s, b)(\tau, \tau) \\
& +A_{5}(s, b)\{\tau, \tau\}+\frac{3 A_{1}}{4}(s, t)\left(\nu_{\tau}, \tau\right) \\
& +A_{5}(c, t)\{\tau, \tau\}+\frac{3 A_{1}}{4}(c, t)\left(\nu_{\tau}, \nu_{\tau}\right)+\text { H.c. },
\end{aligned}
$$

where, following the notation introduced in Ref. [51], we denote

$$
(x, y) \equiv \bar{x}_{L} \gamma^{\mu} y_{L} ; \quad\{x, y\} \equiv \bar{x}_{R} \gamma^{\mu} y_{R} \quad \forall x, y .
$$

Note that $\mathcal{H}^{\mathrm{NP}}$ is expressed in terms of weak eigenstates involving the second- and third-generation quark fields
TABLE I. Benchmark regions to study $(s, b)(\tau, \tau)$ and $(s, b)\{\tau, \tau\}$ operators in the $\left(\mu^{ \pm} \tau^{\mp}\right)$ pair $+b$-jet final state, based on Ref. [52].

\begin{tabular}{lcccccc}
\hline \hline Observables & Set X & Set Y & Set A & Set B & Set C \\
\hline $\operatorname{Br}\left(B_{s} \rightarrow \tau \tau\right)<6.8 \times 10^{-3}$ & $\checkmark$ & $\checkmark$ & $\checkmark$ & $\checkmark$ & $\checkmark$ \\
$3 \sigma$ contour around & $\checkmark$ & $x$ & $x$ & $x$ & $\times$ \\
$A_{1} \approx-3.8, A_{5} \approx-2.3$ & & & & & \\
\hline \hline
\end{tabular}

$\left(Q_{2 L}\right.$ and $\left.Q_{3 L}\right)$ but only the third-generation leptons $\left(L_{3 L}\right)$. While the quark fields would be affected by the usual CKM mixing, in the leptonic sector, the weak eigenstates can be related to the mass eigenstates through a further field rotation [50,51]. This, of course, would induce direct lepton flavor violation. The magnitude of this mixing, as deduced phenomenologically $[50,51]$, is, however, small and was perfectly consistent with $\operatorname{Br}\left(B^{+} \rightarrow K^{+} \mu^{ \pm} \tau^{\mp}\right)<$ $4.8 \times 10^{-5}$ (at $90 \%$ C.L.) [67]. Indeed, it also easily satisfies the recently quoted $95 \%$ C.L. upper bound of $\operatorname{Br}\left(B_{s} \rightarrow \tau^{ \pm} \mu^{\mp}\right)<4.2 \times 10^{-5}$ [68]. This very smallness of the mixing allows us to neglect it altogether and concentrate on the operator $(s, b)(\tau, \tau)$ and $(s, b)\{\tau, \tau\}$ alone. $^{3}$

If an ultraviolet-complete origin of Eq. (10) is desired, an individual term could be parametrized as

$$
X(a, b)(c, d)=\frac{\lambda_{1}^{*} \lambda_{2}}{2 M^{2}}(a, b)(c, d)
$$

where $M$ is the mass of the integrated-out field and $\lambda_{i}$ are some dimensionless couplings, bounded from perturbativity by $\lambda^{2} /(4 \pi)^{2} \leq \mathcal{O}(1)$. The mediator, for example, might be a leptoquark or a $Z^{\prime}$ with flavor-changing couplings. This inequality, along with the requirement of reproducing the requisite $A_{i}$, would determine the ranges allowed to $\lambda_{i}$ and $M$. We, however, eschew any assumption as to the UV-completion, resolutely choosing to be agnostic as to the origin of the $A_{i}$.

While Refs. [51,52] do zero in on "best-fit" points in the parameter space, ${ }^{4}$ note that the exact location of the same is dependent on the accumulation of more data, and, indeed, even the very recent measurements would change it to an extent. Consequently, we investigate the LHC signal for a variety of points, though laying special emphasis to the best-fit point of Ref. [52], namely, $A_{1} \approx-3.8$ and $A_{5} \approx-2.3$, and consider several benchmark regions as listed in Table I.

\footnotetext{
${ }^{3}$ It might be argued that, on inclusion of further quantum corrections, this operator can adversely affect the $B_{s}-\bar{B}_{s}$ mixing. This issue has been adequately addressed in Ref. [51].

${ }^{4}$ The best-fit values were obtained under the assumption of flavor mixing, but our analysis is independent of the mixing angle. Note, too, that the best-fit values of Refs. [51,52] are very similar.
} 
TABLE II. Signal cross section of $\left(\mu^{ \pm} \tau^{\mp}\right)$ pair $+b$-jet after the selections at $13 \mathrm{TeV} p$ - $p$ collision at some benchmark points.

\begin{tabular}{lllccccccc}
\hline \hline Set & $\left|A_{1}\right|$ & $\left|A_{5}\right|$ & $\sigma(\mathbf{S 1})(\mathrm{fb})$ & $\sigma(\mathbf{S 2})(\mathrm{fb})$ & Set & $\left|A_{1}\right|$ & $\left|A_{5}\right|$ & $\sigma(\mathbf{S 1})(\mathrm{fb})$ & $\sigma(\mathbf{S 2})(\mathrm{fb})$ \\
\hline Y1 & 4.5 & 3.0 & 44.52 & 34.72 & A1 & 3.0 & 1.5 & 10.69 & 8.34 \\
Y2 & 4.5 & 3.8 & 70.85 & 55.41 & A2 & 2.5 & 1.5 & 6.54 & 5.12 \\
Y3 & 4.0 & 3.0 & 38.95 & 30.17 & A3 & 2.0 & 1.5 & 5.67 & 4.39 \\
Y4 & 4.0 & 4.0 & 80.32 & 62.66 & A4 & 1.5 & 1.5 & 4.24 & 3.31 \\
X1 & 3.8 & 2.3 & 22.78 & 17.77 & B1 & 2.0 & 1.0 & 3.36 & 2.67 \\
X2 & 3.8 & 3.0 & 35.6 & 27.84 & B2 & 1.5 & 1.0 & 2.31 & 1.8 \\
X3 & 3.8 & 4.0 & 72.39 & 55.83 & B3 & 1.0 & 1.0 & 1.23 & 0.98 \\
Y5 & 3.5 & 2.0 & 16.94 & 13.21 & C1 & 1.0 & 0.5 & 0.81 & 0.63 \\
Y6 & 3.5 & 3.0 & 35.65 & 27.8 & C2 & 0.5 & 0.5 & 0.22 & 0.17 \\
Y7 & 3.0 & 2.3 & 18.70 & 14.63 & C3 & 0.1 & 0.5 & 0.03 & 0.05 \\
Y8 & 3.0 & 3.0 & 31.13 & 24.09 & C4 & 0.05 & 0.5 & 0.02 & 0.01 \\
\hline \hline
\end{tabular}

We consider two factors while defining the regions: One is the $3 \sigma$ contour around the best-fit point as obtained in Ref. [52], and another is the current 95\% C.L. limit on $\operatorname{Br}\left(B_{s} \rightarrow \tau \tau\right)<6.8 \times 10^{-3}$ [69]. All the benchmark points satisfy the latter limit. Set $X$ includes points inside the $3 \sigma$ contour keeping $\left|A_{1}\right|$ fixed at 3.8. Set $Y$ represents points just outside the $3 \sigma$ contour around the best fit. Set $\mathrm{A}$, set $\mathrm{B}$, and set C constitute regions with smaller values of $\left|A_{1}\right|$ and $\left|A_{5}\right|$ and, hence, represent more conservative choices, in the context of both low-energy observables as well as LHC signals. The exact locations of the points are detailed in Table II in the next section, where we study the corresponding collider signals originating from $(s, b)(\tau, \tau)$ and $(s, b)\{\tau, \tau\}$.

\section{COLLIDER STUDY OF $\left(\boldsymbol{\mu}^{ \pm} \boldsymbol{\tau}^{\mp}\right)$ PAIR AND A $b$-JET AT $\sqrt{\hat{s}}=13 \mathrm{TeV}$}

The signature of our interest, namely, a $\mu^{ \pm} \tau^{\mp}$ pair accompanied by a $b$-jet, originates from the operators $(s, b)(\tau, \tau)$ and $(s, b)\{\tau, \tau\}$ in Eq. (10). The requirement of one additional $b$-jet with an opposite sign lepton pair reduces the SM background significantly. The muon, for the signal events, emanates from the decay of a $\tau$. While a direct production of a $\mu^{ \pm} \tau^{\mp}$ pair is possible if the aforementioned lepton mixing is nonzero, the very smallness of the corresponding angle renders this channel to a very subdominant role. ${ }^{5}$ Consequently, we neglect the mixing altogether, even though it is relevant to explain the anomalies. The final states $\tau^{ \pm} \tau^{\mp} b s$ and $\tau^{ \pm} \tau^{\mp} b$ can be produced from $g-g$ fusion and $g-s$ fusion, respectively, in a $p-p$ collision, as shown in Fig. 1, and both the processes are considered in our analysis. In the next section, we determine the sensitivity of the LHC in the recently concluded

\footnotetext{
${ }^{5}$ If one considers flavor mixing, there can be three signatures: $\left(\mu^{ \pm} \mu^{\mp}\right)$ pair $+b$-jet, $\left(\mu^{ \pm} \tau^{\mp}\right)$ pair $+b$-jet, and $\left(\tau^{ \pm} \tau^{\mp}\right)$ pair $+b$-jet, each with effective coupling as a function of the flavor mixing angle. The signature $\left(\mu^{ \pm} \mu^{\mp}\right)$ pair $+b$-jet has been studied in detail recently [53].
}

run as well as in the forthcoming one for the model parameters, $A_{i}$. Towards this, we analyze the signal for each of the benchmark regions as listed in Table I.

Given the preferred size of the four-fermi couplings $A_{i}$, the channel $\left(\tau^{ \pm} \tau^{\mp}\right)$ pair $+b$-jet has a large production cross section, even if we demand that one of the $\tau$ 's decay into a $\mu$ (with a branching fraction of 0.174). On the other hand, owing to the smallness of the lepton-mixing angle, the cross section for direct production, from $g-g$ or $g-s$ fusion, of $\mu^{ \pm} \tau^{\mp}+b$-jet is very small. In the four-fermi limit, the production cross section would depend only on the couplings $A_{i}$ with the subprocess cross section scaling simply as $\hat{s} A_{i}^{2}$, where $\sqrt{\hat{s}}$ is the subprocess center-of-mass energy. This, of course, is moderated by the $\sqrt{\hat{s}}$-dependent parton flux. However, if an ultraviolet-complete theory is considered instead, the dependence on $\hat{s}$ and the mediator mass scale $M$ is more complicated and depends on the precise nature of the completion (for example, a $Z^{\prime}$-like theory would admit the possibility of a resonance, while a leptoquarklike theory would have only $t$-channel propagators). In addition, the phase space distributions would differ as well. However, for a mass-scale $M$ that is larger than a few $\mathrm{TeVs}$, these differences quickly subside primarily on account of the relevant parton fluxes falling quickly with $\sqrt{\hat{s}}$. Not only does this result in a suppression of the fraction of events that could potentially be sensitive to a possible resonance, but any such resonance would also be relative wide one, given the preferred values for the $A_{i}$.

The new physics here is simple enough to permit an analytic calculation, which, when followed by a simplistic simulation, yields rather robust results. Nonetheless, we also implement the effective theory model in Feynrules $[70,71]$ and generate signal events, at the leading order, uniformly throughout the parameter space with MadGraph5_aMC@NLO (v2.2.1) [72] interfaced with PYTHIA [73]. For this, we use the NNPDF23LO1 [74] parton distributions with the five-flavor scheme. We kept the factorization scale fixed at $m_{T}^{2}$ after $k_{T}$ clustering of the event. On varying the factorization scale 

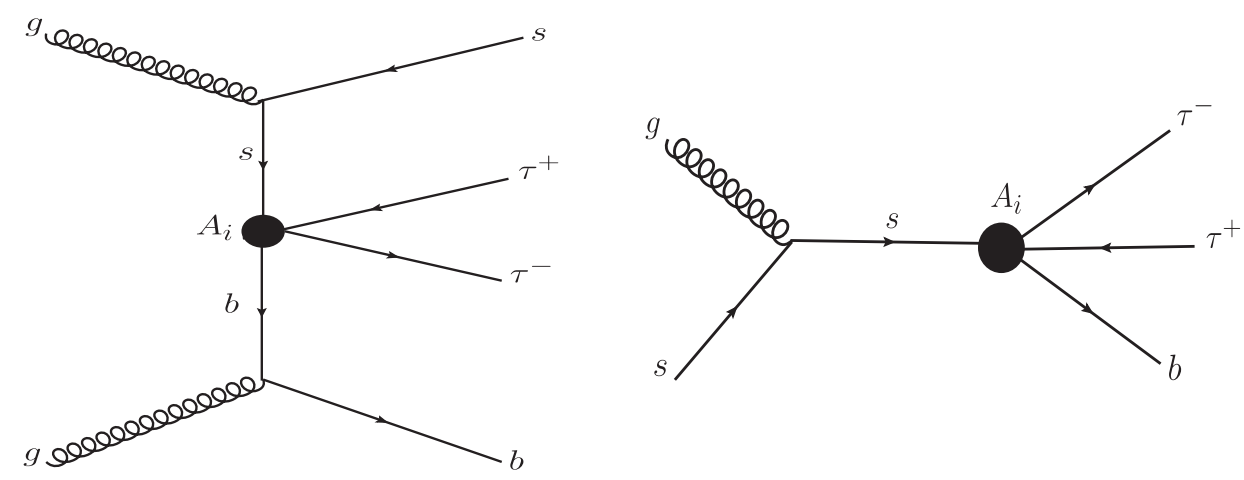

FIG. 1. (Left) Feynman diagram for the production of two $\tau^{\prime}$ s in association with one $b$-jet in $g$ - $g$ fusion $\left[g g \rightarrow \tau^{ \pm} \tau^{\mp} b(s)\right]$ and (right) in $g-s$ fusion $\left[g s \rightarrow \tau^{ \pm} \tau^{\mp} b\right]$. In both figures, the solid blob represents the four-point vertex with effective couplings $A_{i}$.

between $m_{T}^{2} / 4$ and $4 m_{T}^{2}$ and, similarly, scanning over different parton distribution sets, the uncertainty in the LO signal cross section is found to be less than $16 \%$ and $10 \%$, respectively, for the entire range of the parameter space. As for the NLO corrections to the SM, the calculation thereof for a scattering process with a multibody final state such as ours, and especially in an effective theory, is a very arduous task and beyond the scope of the present work. An intelligent estimate can be made nonetheless, by realizing that the effective Hamiltonian of Eq. (10) is most easily obtained starting from a theory with a flavor-changing $Z^{\prime}$ or one with scalar leptoquarks. The $K$ factor for the former is about $1.3[75,76]$, while that for the latter is in the range 1.3-1.4 [77]. It should be appreciated, though, that the relevant couplings considered in said references are not exactly what are needed for the present case. Nevertheless, it stands to reason that the exact $K$ factor should not be wildly different from those quoted above. In other words, the higher-order corrections are expected to increase the signal cross sections. We, however, adopt a conservative standpoint in choosing not to include this enhancement.

The signal comprises two processes, namely, $g s \rightarrow$ $\tau^{ \pm} \tau^{\mp} b$ and $g g \rightarrow \tau^{ \pm} \tau^{\mp} b s$, and only those events are selected wherein one tau decays leptonically to muon, resulting in a $\left(\mu^{ \pm} \tau^{\mp}\right)+b$-jet final state. The events are passed through DELPHES 3 [78], in order to incorporate detector effects and apply reconstruction algorithms. Jets are reconstructed using the anti- $k_{T}$ algorithm in FastJet [79]. For muon isolation, we have required $\Delta R \geq 0.4$ and $p_{T}>1 \mathrm{GeV}$. For calorimetric (tracking) isolation, we require the corresponding momentum parameter to be $0.14(0.15)$ times the $p_{T}$. This ensures that the muons are well isolated from other objects. Tau leptons are reconstructed through their hadronic decays, and we demand that $\Delta R \geq 0.4$ and $p_{T}>10 \mathrm{GeV}$ for the reconstruction. In DELPHES, the tau-tagging efficiency is considered to be 0.6 , and tau misidentification (from gluons and quarks) probability is 0.001 . In this analysis, jets are required to have $p_{T}$ greater than $30 \mathrm{GeV}$ and $\eta(b)<4.7$ and must be separated from the selected leptons by $\Delta R \geq 0.5$. For tagging the $b$-jets, we used a $b$-tagging module inside DELPHES with $70 \%$ working efficiency. The probability of mistagging a charm as a $b$-jet is $10 \%$, while for the other quarks and gluons it is $0.1 \%$ or less.

The backgrounds for this channel can be classified into two categories. The irreducible backgrounds arise mainly from $t \bar{t}$, single top $(W t)$, and diboson $\left(W^{+} W^{-}, W Z\right.$, and $Z Z$ ) production, whereas the $t \bar{t} W$ and $t \bar{t} Z$ contributions are very small. The major contributions to the reducible background arise from $W+$ jets, $Z / \gamma+$ jets, and other QCD multijet processes, where jets may be misidentified as leptons. The probability of a jet to be misidentified as a lepton is taken as a module inside DELPHES [80], as a function of $p_{T}$ and $\eta$ of the jet. All background events are generated using MadGraph, and the cross sections are taken up to NLO and up to NNLO in some cases (see Ref. [81] and references within). Most of the backgrounds will be reduced by the requirement of one $b$-jet and strong isolation selection among the opposite sign leptons.

As we shall see later, the cuts we propose are very effective in suppressing the large background (from a host of SM processes) and, thereby, in increasing the signalto-noise ratio. To eliminate large errors in background modeling, we effect a comparison with an actual experimental study. In particular, the CMS Collaboration has performed a search [66] for a singly produced thirdgeneration scalar leptoquark decaying to a tau lepton and bottom quark in proton-proton collisions at $13 \mathrm{TeV}$ which includes the final state $\left(\mu^{ \pm} \tau^{\mp}\right)$ pair $+b$-jet. The selections we imposed can be summarized as follows: Exactly one each of a $\tau$ and a $\mu$ with these being oppositely charged and with a single $b$-jet, satisfying the following.

\section{Selection S0.-}

$$
\begin{aligned}
p_{T}(\mu) & >50 \mathrm{GeV}, \quad p_{T}(\tau)>50 \mathrm{GeV}, \quad p_{T}(b)>50 \mathrm{GeV}, \\
\eta(\mu) & <2.4, \quad \eta(\tau)<2.5, \quad \eta(b)<2.4 \\
\Delta R(\mu, \tau) & >0.5, \quad \Delta R(\mu, b)>0.5 .
\end{aligned}
$$

Selection S0 constitutes essentially the basic cuts on different variables. The cuts on $\Delta R$ are placed to ensure that 

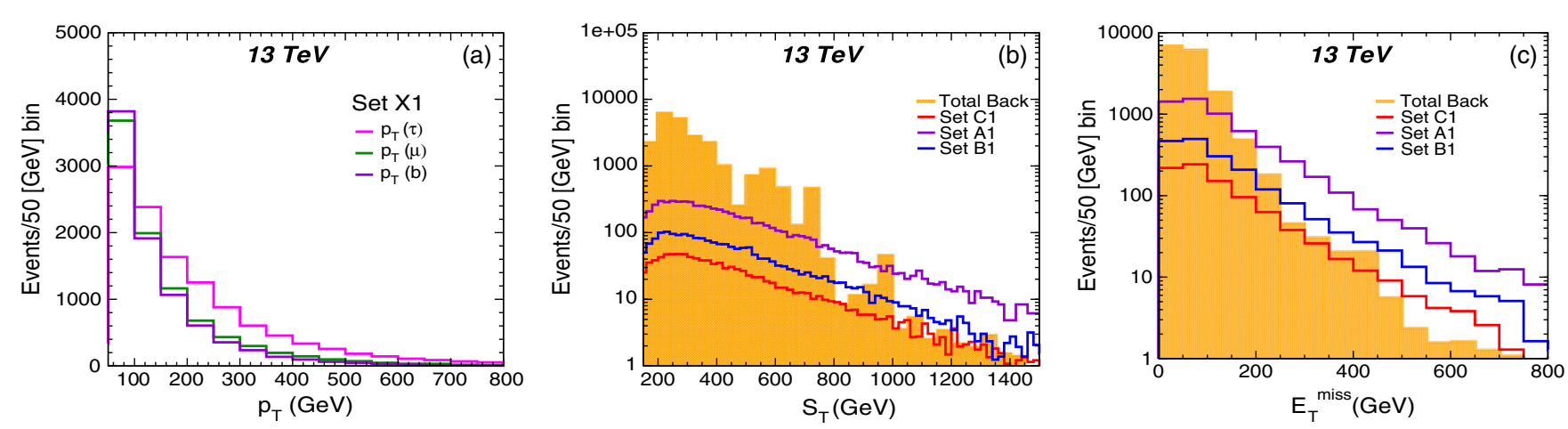

FIG. 2. (a) $p_{T}$ distributions of the signal $\left(\mu^{ \pm} \tau^{\mp}\right)$ pair $+b$-jet. (b) $S_{T}=p_{T}(\mu)+p_{T}(\tau)+p_{T}$ (b) distribution for the signal and total background. (c) $E_{T}^{\text {miss }}$ for the signal and total background. All plots are done after after selection S0. The signals are normalized for sets $\mathrm{X} 1\left(\left|A_{1}\right|=3.8\right.$ and $\left.\left|A_{5}\right|=2.3\right), \mathrm{C} 1\left(\left|A_{1}\right|=1.0\right.$ and $\left.\left|A_{5}\right|=0.5\right), \mathrm{A} 1\left(\left|A_{1}\right|=3.0\right.$ and $\left.\left|A_{5}\right|=1.5\right)$, and $\mathrm{B} 1\left(\left|A_{1}\right|=2.0\right.$ and $\left.\left|A_{5}\right|=1.0\right)$. Events are weighted at $120 \mathrm{fb}^{-1}$.

the muon is well isolated from the tau and the $b$-jet. We have plotted the respective $p_{T}$ distributions for the tau, the muon, and the $b$-jet for the signal corresponding to a representative point in the parameter space in Fig. 2(a). As already discussed, the hard interaction being a four-fermi one, the production cross sections, typically, grow with the partonic center-of-mass energy (modulo the suppression due to the effective flux). Furthermore, with the cross sections slightly favoring large angle scattering over small angle, each of the two $\tau$ 's as well as the $b$-jet tend to have a sufficiently large $p_{T}$. While the $\tau^{ \pm}$have essentially identical distributions, the $b$ has a softer component that arises from the $g g$-initiated process. The $\mu$, while having considerable $p_{T}$, is softer than the $\tau$, being only a descendant of the second $\tau$. Given this, it is profitable to impose a stronger cut in terms of the variable $S_{T}$, which is the scalar sum of the $p_{T}$ of the final state particles. The distribution is shown in Fig. 2(b) for both the signal and the background. The signal is associated with a relatively modest $E_{T}^{\text {miss }}$, as seen from Fig. 2(c). As in Ref. [66], a soft cut on $M_{\mu \tau}$ in $\mathbf{S 1}$ is essential to reduce the background coming from the $t \bar{t}$ and single top production. To summarize, the following set of cuts are used, as in Ref. [66].

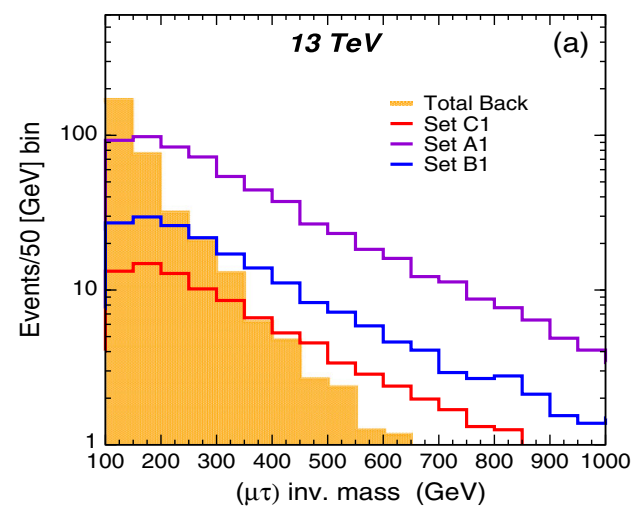

\section{Selection S1.-}

$$
\begin{aligned}
S_{T}=p_{T}(\mu)+p_{T}(\tau)+p_{T}(b) & >500 \mathrm{GeV}, \\
M_{(\mu, \tau)} & >85 \mathrm{GeV} .
\end{aligned}
$$

The distributions at this stage are depicted in Fig. 3. It is worthwhile to point out that our background profile shape agrees very well with the CMS results [66], while we exceed them in total count by about $5 \%$. In other words, our background determination is very robust.

At this stage, we propose the following additional selections to improve the signal efficiency.

Selection S2.-

$$
\begin{aligned}
E_{T}^{\mathrm{miss}} & <230 \mathrm{GeV}, \\
M_{(b, \mu, \tau)} & >600 \mathrm{GeV} .
\end{aligned}
$$

These particular choices need some explaining. While the major background process $(t \bar{t})$ has a pair of neutrinos associated with the hard process, the signal has none (and the $E_{T}^{\text {miss }}$ arises from cascade decays of the hadron as well as mismeasurements). Consequently, a strong upper cut on

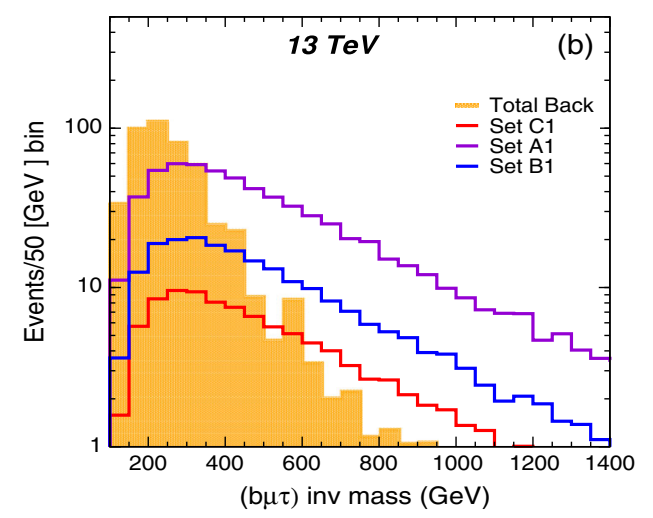

FIG. 3. The behavior of the (a) two-body and (b) three-body invariant mass of distribution of the signal $\left(\mu^{ \pm} \tau^{\mp}\right)$ pair $+b$-jet for $\mathrm{C} 1, \mathrm{~A} 1$, and B1 (defined in Table II) and the total background is shown after the S1 cut. Events are weighted at $120 \mathrm{fb}^{-1}$. 
TABLE III. Background cross sections after several selections at $13 \mathrm{TeV} p$ - $p$ collision.

\begin{tabular}{lcc}
\hline \hline Background & $\sigma(\mathbf{S 1})(\mathrm{fb})$ & $\sigma(\mathbf{S 2})(\mathrm{fb})$ \\
\hline$t \bar{t}$ & 5.715 & 2.78 \\
$W t$ & 1.132 & 0.502 \\
$W+$ jets & 0.275 & 0.241 \\
$Z / \gamma+$ jets & 0.076 & 0.038 \\
Diboson & 0.014 & 0.0017 \\
QCD + multijets & 0.0624 & 0.016 \\
Total & 7.276 & 3.6 \\
\hline \hline
\end{tabular}

$E_{T}^{\text {miss }}$ would be expected to be useful. In the present case, though, the backgrounds from the single top and $W+$ jets processes are not negligible, and these are not reduced overwhelmingly by the imposition of such a cut. As a result. the loss of background resulting from a stronger upper cut on $E_{T}^{\text {miss }}$ does not compensate enough the attendant loss in the signal strength. Hence, we impose only a loose upper cut of $E_{T}^{\text {miss }}<230 \mathrm{GeV}$ in $\mathbf{S 2}$. While the entire preceding discussion may seem infructuous in view of Fig. 2(c), it should be viewed in conjunction with the cut on the three-body invariant mass $M_{(b, \mu, \tau)}$, which plays a major role in preferentially reducing the backgrounds. As Fig. 3(b) demonstrates, a strong cut on this variable is expected to improve the signal-to-noise ratio. Indeed, once this is imposed (as in $\mathbf{S 2}$ ), it turns out that it is an upper restriction on $E_{T}^{\text {miss }}$ that is more useful rather than a lower cut. Moreover, these particular set of cuts in $\mathbf{S 2}$ are useful when signal cross section is comparatively small for small $A_{i}$. The signal cross sections after the cuts are given in Table II for different benchmark points of $A_{1}$ and $A_{5}$. We have analyzed the signal as a function of $A_{1}$ and $A_{5}$, in different regions and show the variation in the signal cross sections.

The cut flow of the backgrounds with the selections is demonstrated in Table III. Even after the selection S2, the majority of the total background comes from $t \bar{t}$ and single top production. Also note that the signal retains a reasonable number of events when passed through S2. The background is notably smaller for the $\mathbf{S 2}$ selection as compared to that for $\mathbf{S 1}$. Even though the discovery and exclusion limits can be obtained with both $\mathbf{S 1}$ and $\mathbf{S 2}$, and their behavior is comparable, in the next section we show the result when events are selected through $\mathbf{S 2}$.

\section{RESULTS}

As the analysis of the preceding section shows, it is indeed possible to exclude much of the parameter space favored by the resolution of the $B$ anomalies and even contemplate discovery. Rather than restrict ourselves to simplistic signal-to-noise estimations, we consider, instead, a slightly more sophisticated statistical test. Towards this end, let us define the null hypothesis as the set of events being composed entirely of the background (irreducible or instrumental). This is to be tested against the alternative hypothesis, which includes both the background as well as the sought after signal. To summarize the outcome of such a search, one quantifies the level of agreement of the observed data with a given hypothesis by computing the $p$ value. This $p$ value can be converted into an equivalent significance, $Z_{\mathrm{dis}}$, for a Gaussian distributed variable. The exact formulation is summarized in the Appendix, with the $5 \sigma$ discovery significance $\left(Z_{\mathrm{dis}}=5\right)$ and $95 \%$ C.L. exclusion limit $\left(Z_{\text {exc }}=1.645\right)$ being given by Eqs. (A1) and (A4), respectively.

We can now consider the discovery and exclusion prospects of this particular channel under study and compute the required integrated luminosity $\left(\mathcal{L}_{\text {int }}\right)$ as a function of model parameters $A_{1,5}$. A straightforward computation, using the signal and background cross sections calculated in the last section, leads to very optimistic results, though. However, the background is not known with a very good precision. To account for this, we include a variance $\Delta_{b}$ in the background while calculating the discovery significance and exclusion from Refs. [82-84] (see $[85,86]$ for more detail). With the current LHC data and the data that LHC will take in the future, the systematic experimental uncertainties in the estimation of the SM backgrounds are expected to be reduced significantly, and the detector response is also expected to be better in future. For example, by comparing Refs. [87,88], one can see find how the systematic uncertainty has reduced so far at the LHC. So, additionally, we also assume in Eqs. (A1) and (A4) that a part of the systematic uncertainty falls as $1 / \sqrt{\mathcal{L}_{\text {int }}}$.

In Fig. 4 , we plot the discovery significance $\left(Z_{\mathrm{dis}}\right)$ for different values of $A_{1,5}$ (note that the signal cross section and, hence, the significance are essentially independent of the sign of the Wilson coefficients) as a function of the integrated luminosity $\left(\mathcal{L}_{\text {int }}\right)$ for some benchmark points. It is evident from Fig. 4(b) that, for set X, a small value of $\mathcal{L}_{\text {int }}$ is required to achieve $5 \sigma$ discovery significance. This is because of the large signal cross section, as can be seen from Table II. For the best-fit scenario (X1), $Z_{\text {dis }} \geq 5$ can be achieved with $\mathcal{L}_{\text {int }} \sim 2 \mathrm{fb}^{-1}$, even with a $50 \%$ uncertainty in the background estimation. For a wide range of values of $A_{1}$ and $A_{5}, Z_{\text {dis }}>5$ is also achievable with the luminosity achieved so far at the LHC, as can be envisaged from Fig. 4. The significance, as expected, depends on the signal cross section, which, in turn, depends on $A_{1}$ and $A_{5}$. Thus, with lower values of these couplings (such as $\mathrm{C} 3$ and $\mathrm{C} 4$ ), one has to wait for a larger $\mathcal{L}_{\text {int }}$. Overall, we find that a large region of the model parameter space can be probed with $5 \sigma$ or higher significance with current LHC data.

In Fig. 5, we show the variation of the model parameters $A_{1}$ and $A_{5}$ as a function of $\mathcal{L}_{\text {int }}$ that is needed to get $Z_{\text {dis }}=5$. The horizontal black dashed lines represent the 

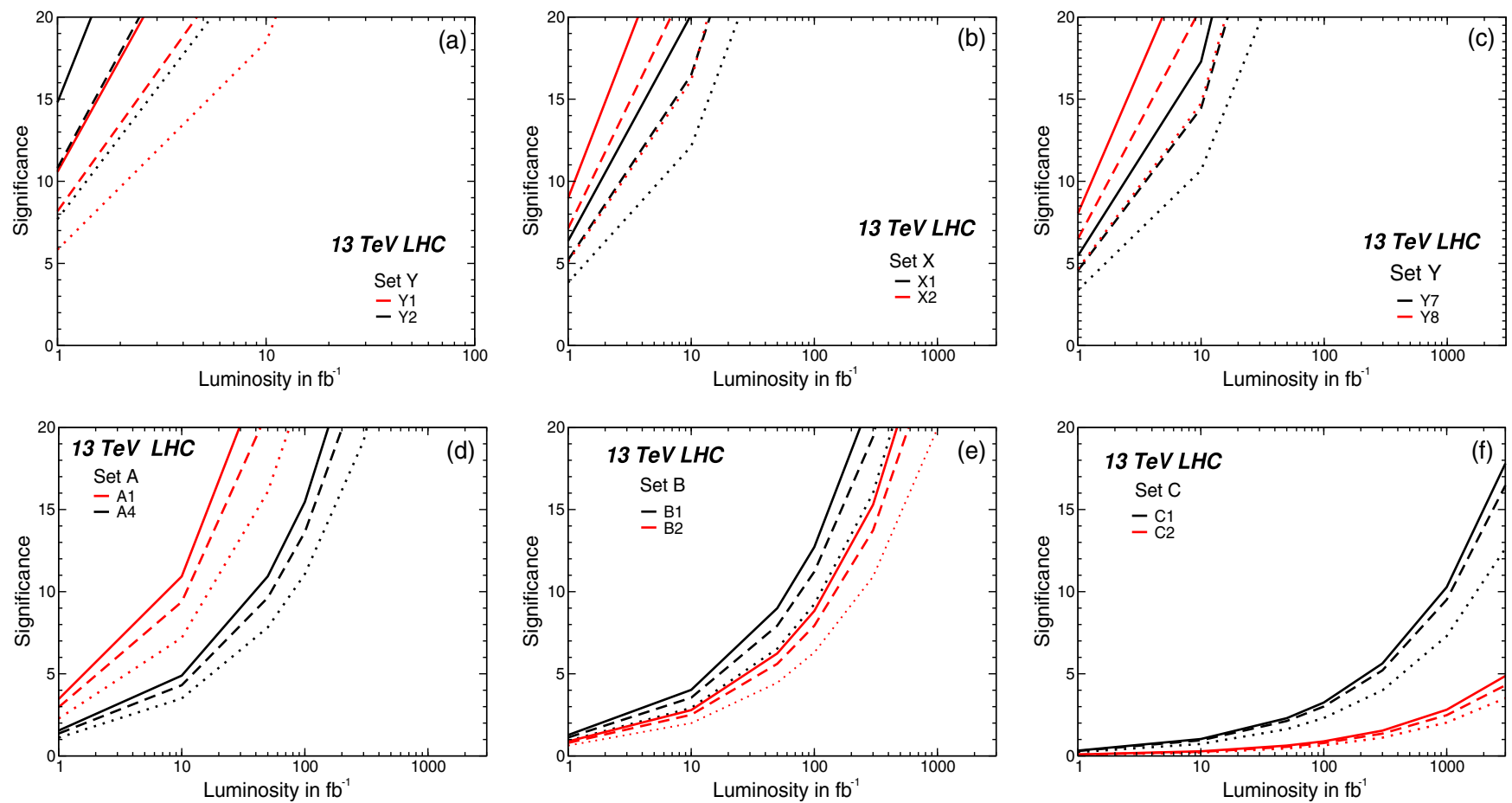

FIG. 4. The discovery significance $\left(Z_{\text {dis }}\right)$ as a function of the integrated luminosity $\left(\mathcal{L}_{\text {int }}\right)$. Solid, dashed, and dotted lines represent $0 \%$, $25 \%$, and $50 \%$ uncertainty in the background events, respectively for different sets as mentioned in the text. Events are selected by $\mathbf{S 2}$. The best-fit values are $\left|A_{1}\right|=3.8$ and $\left|A_{5}\right|=2.3$, represented by set $\mathrm{X} 1$ in (b).

current $\mathcal{L}_{\text {int }}$ at the LHC; $150 \mathrm{fb}^{-1}$ for each of the two experiments ATLAS and CMS and $300 \mathrm{fb}^{-1}$ combined. In Fig. 5, we show this variation for set B (left), set A (middle), and set $\mathrm{X}$ (right); the values of $A_{1}$ and $A_{5}$ are chosen in such a way as to satisfy all the low-energy constraints, as mentioned before. If the values of $A_{1}$ and $A_{5}$ lie close to their best-fit values, it is evident from Fig. 5 (middle and right) that even a small $\mathcal{L}_{\text {int }}$ is sufficient to either validate or falsify the model, which should be the case once the present dataset is fully analyzed. Figure 5 (left) shows that with smaller values of $\left|A_{1}\right|$ and $\left|A_{5}\right|$ (set B) it is likely to reach $5 \sigma$ significance with current LHC data, even if the uncertainty in the background estimation is 50\% or more. For set $\mathrm{C}$, much higher luminosity is required for $5 \sigma$ discovery; hence, we refrain from showing the corresponding plots.

In Fig. 6, we show the exclusion limits on $A_{1}$, keeping $A_{5}$ as a parameter, in terms of $\mathcal{L}_{\text {int }}$. The plots, from left to right, are for sets $\mathrm{C}, \mathrm{B}$, and $\mathrm{A}$, respectively. We do not display the corresponding plots from sets $\mathrm{X}$ and $\mathrm{Y}$, as the required luminosity is quite small, as discussed before. Overall with the current LHC data, 95\% C.L. exclusion limits can be set in a large region of the model parameter space including the small values of $A_{1}$ and $A_{5}$.
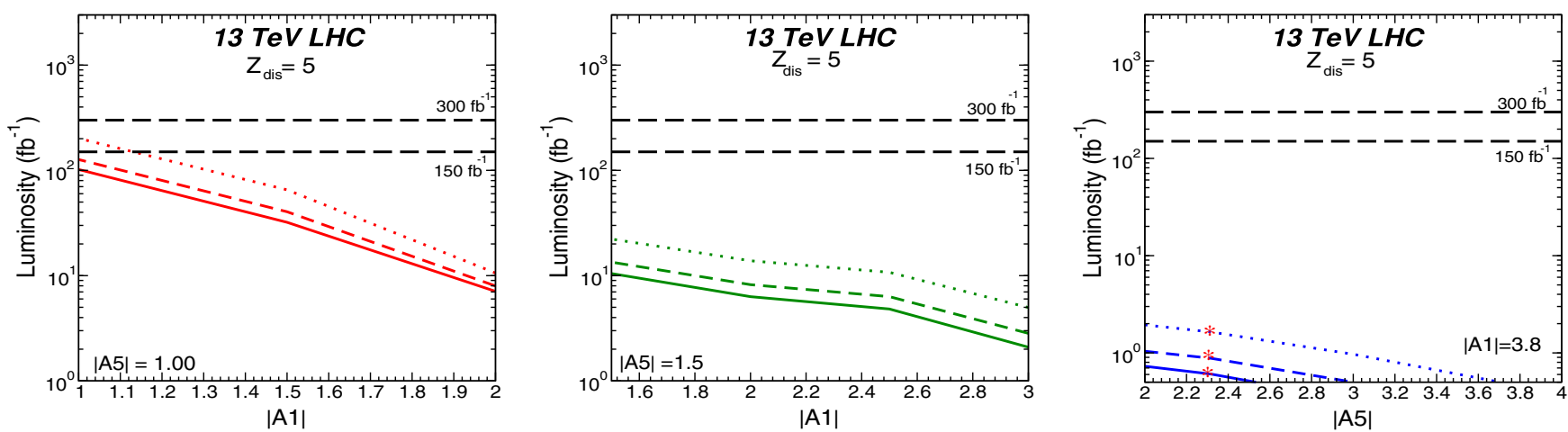

FIG. 5. The $5 \sigma$ discovery reach at the $13 \mathrm{TeV}$ LHC in the $\left(\mu^{ \pm} \tau^{\mp}\right)+b$-jet channel as a function of $\mathcal{L}_{\text {int }}$ and $A_{1}, A_{5}$ (in TeV ${ }^{-2}$ ). Solid, dashed, and dotted lines represent $0 \%, 25 \%$, and 50\% uncertainty in the background events, respectively. Events are selected by $\mathbf{S 2}$. The best-fit values are $\left|A_{1}\right|=3.8$ and $\left|A_{5}\right|=2.3$, represented by red stars. 

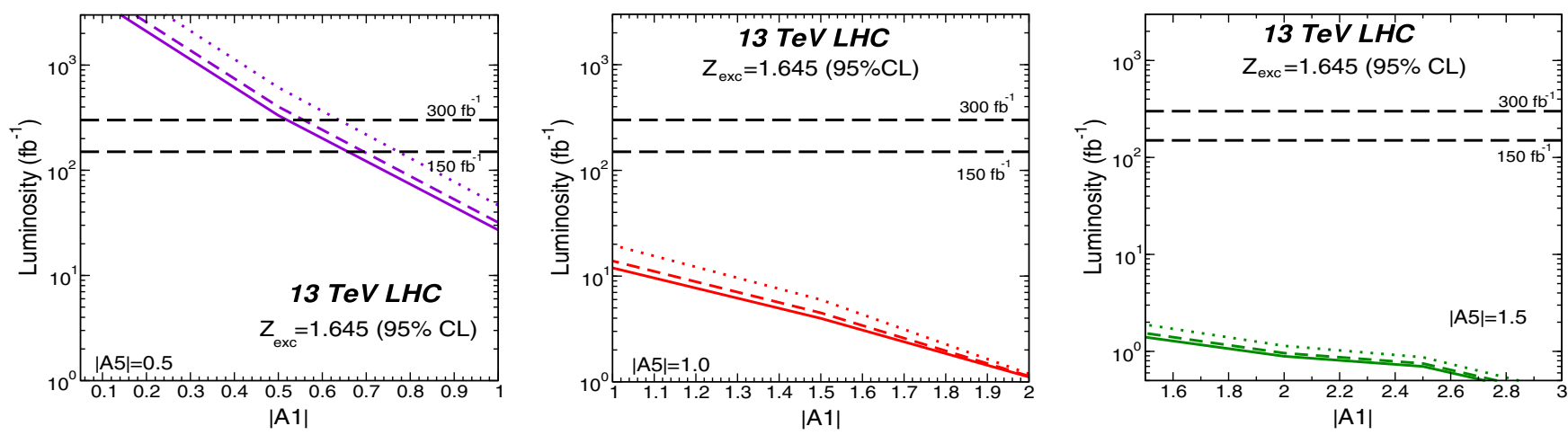

FIG. 6. The 95\% C.L. exclusion limits at the $13 \mathrm{TeV}$ LHC in the $\left(\mu^{ \pm} \tau^{\mp}\right)+b$-jet channel as a function of $\mathcal{L}_{\text {int }}$ and $A_{1}, A_{5}$ (in $\mathrm{TeV}^{-2}$ ). Solid, dashed, and dotted lines represent $0 \%, 25 \%$, and 50\% uncertainty in the background events, respectively. Events are selected by $\mathbf{S 2}$.

\section{OUTLOOK}

The not-too-insignificant anomalies in semileptonic $B$ meson decays point towards some new physics that violate lepton flavor universality. One interesting option is to consider effective dimension- 6 operators of the form $a_{i j}\left(\bar{b} \Gamma_{i} s\right)\left(\bar{\tau} \Gamma_{j} \tau\right)$, where $\Gamma_{i}$ are operators in the Dirac space. The charged-current counterpart of this operator-arising automatically when $S U(2)_{L} \otimes U(1)_{Y}$ symmetry is imposed-may explain the $R(D)$ and $R\left(D^{*}\right)$ anomalies, while this operator itself, aided by lepton flavor mixing, may lead to a possible explanation of the $R_{K}$ and $R_{K^{*}}$ results. Without the knowledge of the ultimate ultravioletcomplete theory, the most prudent way to explore the parameter space for new physics is in terms of the Wilson coefficients. Nominally, these would go as $\lambda^{2} / M^{2}$, where $\lambda$ is some dimensionless coupling and $M$ is the mass of the integrated-out mediator, which may be taken as the scale of new physics.

In this paper, we explored direct signals from such class of operators at the LHC. While probing the structure of the most general set of such four-fermi operators would be difficult, the task has been eased by the analyses of Refs. [50-52], which have shown that said anomalies can be very satisfactorily resolved in terms of just two Wilson coefficients, denoted by $A_{1,5}$. We adopt this simplified structure and also examine how well these can be explored in terms of the integrated luminosity. As long as $M$ is much above the scale being probed by the LHC, whether the new operators are generated through an extra $Z^{\prime}$, or leptoquarks, or some other dynamics, is irrelevant.

The signal that we focused upon is an unlike-charged $\mu-\tau$ pair associated with a $b$-jet, where the muon comes from the leptonic decay of one of the daughter $\tau$ 's. With suitable cuts, one may reduce the SM backgrounds for this signal to a very small level and, thus, have a very good detection prospect, even with just the currently collected data, for values of $A_{1,5}$ preferred by the analyses of Refs. [50-52]. However the values of $A_{1,5}$ are constrained from the nonobservation of $\operatorname{Br}\left(B_{s} \rightarrow \tau \tau\right)$ and, hence, cannot be chosen arbitrarily. Even with an uncertainty in the estimation of the background, the situation looks quite optimistic. For example, notwithstanding the agreement of our background estimation, post $\mathbf{S 2}$, with the experimental results of Ref. [66], let us consider the ramifications of an uncertainty as large as $\sim 50 \%$ in the background estimation. Even for the point $\left(\left|A_{1}\right|,\left|A_{5}\right|\right)=(1.8,1.5)$, somewhat smaller than the best-fit values of these parameters (and, hence, resulting in a smaller cross section) can be probed with $5 \sigma$ significance at $\mathcal{L}_{\text {int }}=20 \mathrm{fb}^{-1}$. Similarly, with the same $\mathcal{L}_{\text {int }}$ and a similar uncertainty in the background, the region of parameter space defined by $\left(\left|A_{1}\right| \geq 1.0,\left|A_{5}\right| \geq 1.0\right)$ can be excluded at $95 \%$ C.L. For even smaller values of $A_{i}$, one requires a significantly larger luminosity; for example, the region $\left(\left|A_{1}\right| \geq 0.8,\left|A_{5}\right| \geq 0.5\right)$ can be excluded at 95\% C.L. with $150 \mathrm{fb}^{-1}$.

The case where both the $\tau$ 's decay hadronically is not so clean as this channel but will be taken up in a subsequent study. We have not taken the lepton flavor mixing between $\mu$ and $\tau$ into account. As has been shown in the literature, the mixing angle is bound to be small $(\sim 0.02)$. While the mixing can directly produce an unlike-sign $\tau-\mu$ pair, the production rate is swamped by the events where one $\tau$ subsequently decays into a muon. Thus, we do not envisage that a study of this nature will shed any light on the mixing angle. This would be better investigated by significantly improving the measurements of lepton-flavor-violating decays such as $B \rightarrow K^{(*)} \mu \tau$ or $B_{s} \rightarrow \tau^{ \pm} \mu^{\mp}$.

\section{ACKNOWLEDGMENTS}

D. C. and A. K. are supported by the Science and Engineering Research Board, India under Grants No. CRG/2018/004889 and No. EMR/2016/001306, respectively. N.K. acknowledges the support from the Indo-French Center for Promotion of Advanced Research (CEFIPRA Project No. 5404-2) and also Dr. D. S. Kothari Postdoctoral scheme (201819-PH/18-19/ 
0013). N. K. also thanks the organizers of "DAE BRNS Symposium-2018" for providing the opportunity to present and discuss the results of this paper. We also thank the referee for useful comments and suggestions.

\section{APPENDIX: DISCOVERY SIGNIFICANCE AND EXCLUSION LIMIT}

The significance for discovery in terms of signal events $(s)$, background events $(b)$, and the uncertainty in the background $\left(\Delta_{b}\right)$ is [82-84]

$$
\begin{aligned}
Z_{\mathrm{dis}}= & {\left[2 \left((s+b) \ln \left[\frac{(s+b)\left(b+\Delta_{b}^{2}\right)}{b^{2}+(s+b) \Delta_{b}^{2}}\right]\right.\right.} \\
& \left.\left.-\frac{b^{2}}{\Delta_{b}^{2}} \ln \left[1+\frac{\Delta_{b}^{2} s}{b\left(b+\Delta_{b}^{2}\right)}\right]\right)\right]^{1 / 2} .
\end{aligned}
$$

If $\Delta_{b}=0$,

$$
Z_{\mathrm{dis}}=\sqrt{2[(s+b) \ln (1+s / b)-s]} .
$$

In the above equation, if $b$ is large, then we obtain the well-known expression

$$
Z_{\mathrm{dis}}=s / \sqrt{b}
$$

For discovery reach, $Z_{\mathrm{dis}} \geq 5$ corresponds to $5 \sigma$ discovery $\left(p<2.86 \times 10^{-7}\right)$. The exclusion limit at a given confidence level (C.L.) is [82-84]

$$
\begin{aligned}
Z_{\mathrm{exc}}= & {\left[2\left\{s-b \ln \left(\frac{b+s+x}{2 b}\right)-\frac{b^{2}}{\Delta_{b}^{2}} \ln \left(\frac{b-s+x}{2 b}\right)\right\}\right.} \\
& \left.-(b+s-x)\left(1+b / \Delta_{b}^{2}\right)\right]^{1 / 2},
\end{aligned}
$$

where

$$
x=\sqrt{(s+b)^{2}-4 s b \Delta_{b}^{2} /\left(b+\Delta_{b}^{2}\right)} .
$$

In the above equation, if $\Delta_{b}=0$,

$$
Z_{\text {exc }}=\sqrt{2(s-b \ln (1+s / b))} .
$$

For a median expected $95 \%$ C.L. exclusion ( $p=0.05$ ), we use $Z_{\text {exc }} \geq 1.645$ for different $\Delta_{b}$.
[1] J. P. Lees et al. (BABAR Collaboration), Measurement of an excess of $\bar{B} \rightarrow D^{(*)} \tau^{-} \bar{\nu}_{\tau}$ decays and implications for charged Higgs bosons, Phys. Rev. D 88, 072012 (2013).

[2] M. Huschle et al. (Belle Collaboration), Measurement of the branching ratio of $\bar{B} \rightarrow D^{(*)} \tau^{-} \bar{\nu}_{\tau}$ relative to $\bar{B} \rightarrow D^{(*)} \ell^{-} \bar{\nu}_{\ell}$ decays with hadronic tagging at Belle, Phys. Rev. D 92, 072014 (2015).

[3] A. Abdesselam et al. (Belle Collaboration), Measurement of the branching ratio of $\bar{B}^{0} \rightarrow D^{*+} \tau^{-} \bar{\nu}_{\tau}$ relative to $\bar{B}^{0} \rightarrow$ $D^{*+} \ell^{-} \bar{\nu}_{\ell}$ decays with a semileptonic tagging method, in Proceedings of the 51st Rencontres de Moriond on Electroweak Interactions and Unified Theories: La Thuile, Italy, 2016 (ARISF, 2016).

[4] S. Hirose et al. (Belle Collaboration), Measurement of the $\tau$ Lepton Polarization and $R\left(D^{*}\right)$ in the Decay $\bar{B} \rightarrow D^{*} \tau^{-} \bar{\nu}_{\tau}$, Phys. Rev. Lett. 118, 211801 (2017).

[5] Y. Sato et al. (Belle Collaboration), Measurement of the branching ratio of $\bar{B}^{0} \rightarrow D^{*+} \tau^{-} \bar{\nu}_{\tau}$ relative to $\bar{B}^{0} \rightarrow$ $D^{*+} \ell^{-} \bar{\nu}_{\ell}$ decays with a semileptonic tagging method, Phys. Rev. D 94, 072007 (2016).

[6] S. Hirose et al. (Belle Collaboration), Measurement of the $\tau$ lepton polarization and $R\left(D^{*}\right)$ in the decay $\bar{B} \rightarrow D^{*} \tau^{-} \bar{\nu}_{\tau}$ with one-prong hadronic $\tau$ decays at Belle, Phys. Rev. D 97, 012004 (2018).

[7] A. Abdesselam et al. (Belle Collaboration), Test of lepton flavor universality in $B \rightarrow K^{*} \ell^{+} \ell^{-}$decays at Belle, arXiv: 1904.02440.
[8] R. Aaij et al. (LHCb Collaboration), Measurement of the Ratio of Branching Fractions $\mathcal{B}\left(\bar{B}^{0} \rightarrow D^{*+} \tau^{-} \bar{\nu}_{\tau}\right) /$ $\mathcal{B}\left(\bar{B}^{0} \rightarrow D^{*+} \mu^{-} \bar{\nu}_{\mu}\right)$, Phys. Rev. Lett. 115, 111803 (2015).

[9] R. Aaij et al. (LHCb Collaboration), Measurement of the Ratio of the $B^{0} \rightarrow D^{*-} \tau^{+} \nu_{\tau}$ and $B^{0} \rightarrow D^{*-} \mu^{+} \nu_{\mu}$ Branching Fractions Using Three-Prong $\tau$-Lepton Decays, Phys. Rev. Lett. 120, 171802 (2018).

[10] R. Aaij et al. (LHCb Collaboration), Test of Lepton Flavor Universality by the measurement of the $B^{0} \rightarrow D^{*-} \tau^{+} \nu_{\tau}$ branching fraction using three-prong $\tau$ decays, Phys. Rev. D 97, 072013 (2018).

[11] R. Aaij et al. (LHCb Collaboration), Test of Lepton Universality Using $B^{+} \rightarrow K^{+} \ell^{+} \ell^{-}$Decays, Phys. Rev. Lett. 113, 151601 (2014).

[12] R. Aaij et al. (LHCb Collaboration), Test of lepton universality with $B^{0} \rightarrow K^{* 0} \ell^{+} \ell^{-}$decays, J. High Energy Phys. 08 (2017) 055.

[13] LHCb Collaboration, Search for lepton-universality violation in $B^{+} \rightarrow K^{+} \ell^{+} \ell^{-}$decays, Technical Reports No. CERN-EP-2019-043 and No. LHCB-PAPER-2019009, CERN, Geneva, 2019.

[14] Y. Amhis et al. (HFLAV Collaboration), Averages of $b$-hadron, $c$-hadron, and $\tau$-lepton properties as of summer 2016, Eur. Phys. J. C 77, 895 (2017).

[15] Y. Amhis et al. (Heavy Flavor Averaging Group), Averages of $b$-hadron, $c$-hadron, and $\tau$-lepton properties as of summer 2014, arXiv:1412.7515. 
[16] https://hflav-eos.web.cern.ch/hflav-eos/semi/summer18/ RDRDs.html.

[17] M. A. Ivanov, J. G. Korner, and P. Santorelli, Semileptonic decays of $B_{c}$ mesons into charmonium states in a relativistic quark model, Phys. Rev. D 71, 094006 (2005).

[18] R. Dutta and A. Bhol, $B_{c} \rightarrow\left(J / \psi, \eta_{c}\right) \tau \nu$ semileptonic decays within the Standard Model and beyond, Phys. Rev. D 96, 076001 (2017).

[19] R. Watanabe, New physics effect on $B_{c} \rightarrow J / \psi \tau \tau \bar{\nu}$ in relation to the $R_{D^{(*)}}$ anomaly, Phys. Lett. B 776, 5 (2018).

[20] G. Hiller and F. Kruger, More model-independent analysis of $b \rightarrow s$ processes, Phys. Rev. D 69, 074020 (2004).

[21] C. Bobeth, G. Hiller, and G. Piranishvili, Angular distributions of $\bar{B} \rightarrow \bar{K} \ell^{+} \ell^{-}$decays, J. High Energy Phys. 12 (2007) 040 .

[22] M. Bordone, G. Isidori, and A. Pattori, On the Standard Model predictions for $R_{K}$ and $R_{K^{*}}$, Eur. Phys. J. C 76, 440 (2016).

[23] B. Capdevila, S. Descotes-Genon, J. Matias, and J. Virto, Assessing lepton-flavour non-universality from $B \rightarrow K^{*} \ell \ell$ angular analyses, J. High Energy Phys. 10 (2016) 075.

[24] N. Serra, R. Silva Coutinho, and D. van Dyk, Measuring the breaking of lepton flavor universality in $B \rightarrow K^{*} \ell^{+} \ell^{-}$, Phys. Rev. D 95, 035029 (2017).

[25] R. Aaij et al. (LHCb Collaboration), Angular analysis and differential branching fraction of the decay $B_{s}^{0} \rightarrow \phi \mu^{+} \mu^{-}$, J. High Energy Phys. 09 (2015) 179.

[26] W. Altmannshofer and D. M. Straub, New physics in $b \rightarrow s$ transitions after LHC run 1, Eur. Phys. J. C 75, 382 (2015).

[27] A. Bharucha, D. M. Straub, and R. Zwicky, $B \rightarrow V \ell^{+} \ell^{-}$in the Standard Model from light-cone sum rules, J. High Energy Phys. 08 (2016) 098.

[28] LHCb Collaboration, CERN Technical Reports No. LHCbCONF-2015-002 and No. CERN-LHCb-CONF-2015-002, 2015.

[29] A. Datta, J. Kumar, and D. London, The $B$ anomalies and new physics in $b \rightarrow s e^{+} e^{-}$, Phys. Lett. B 797, 134858 (2019).

[30] P. Langacker, The physics of heavy $Z^{\prime}$ gauge bosons, Rev. Mod. Phys. 81, 1199 (2009).

[31] A. Falkowski, M. Nardecchia, and R. Ziegler, Lepton flavor non-universality in B-meson decays from a $\mathrm{U}(2)$ flavor model, J. High Energy Phys. 11 (2015) 173.

[32] A. J. Buras and J. Girrbach, Left-handed $Z^{\prime}$ and $Z$ FCNC quark couplings facing new $b \rightarrow s \mu^{+} \mu^{-}$data, J. High Energy Phys. 12 (2013) 009.

[33] J. F. Kamenik, Y. Soreq, and J. Zupan, Lepton flavor universality violation without new sources of quark flavor violation, Phys. Rev. D 97, 035002 (2018).

[34] A. Carmona and F. Goertz, Lepton Flavor and Nonuniversality from Minimal Composite Higgs Setups, Phys. Rev. Lett. 116, 251801 (2016).

[35] M. Chala and M. Spannowsky, Behavior of composite resonances breaking lepton flavor universality, Phys. Rev. D 98, 035010 (2018).

[36] M. Chala, U. Egede, and M. Spannowsky, Searching new physics in rare $B$-meson decays into multiple muons, Eur. Phys. J. C 79, 431 (2019).

[37] D. Beirevi, S. Fajfer, N. Konik, and O. Sumensari, Leptoquark model to explain the $B$-physics anomalies, $R_{K}$ and $R_{D}$, Phys. Rev. D 94, 115021 (2016).
[38] S. Fajfer and N. Konik, Vector leptoquark resolution of $R_{K}$ and $R_{D^{(*)}}$ puzzles, Phys. Lett. B 755, 270 (2016).

[39] M. Blanke and A. Crivellin, $B$ Meson Anomalies in a PatiSalam Model within the Randall-Sundrum Background, Phys. Rev. Lett. 121, 011801 (2018).

[40] R. Barbier et al., R-parity violating supersymmetry, Phys. Rep. 420, 1 (2005).

[41] G. R. Farrar and P. Fayet, Phenomenology of the production, decay, and detection of new hadronic states associated with supersymmetry, Phys. Lett. 76B, 575 (1978).

[42] W. Altmannshofer, P. S. Bhupal Dev, and A. Soni, $R_{D^{(*)}}$ anomaly: A possible hint for natural supersymmetry with $R$-parity violation, Phys. Rev. D 96, 095010 (2017).

[43] R. Alonso, B. Grinstein, and J. Martin Camalich, Lifetime of $B_{c}^{-}$Constrains Explanations for Anomalies in $B \rightarrow D^{(*)} \tau \nu$, Phys. Rev. Lett. 118, 081802 (2017).

[44] D. Bardhan, P. Byakti, and D. Ghosh, Role of tensor operators in $R_{K}$ and $R_{K^{*}}$, Phys. Lett. B 773, 505 (2017).

[45] C. Bobeth, T. Ewerth, F. Kruger, and J. Urban, Analysis of neutral Higgs boson contributions to the decays $B s \rightarrow \ell^{+} \ell^{-}$and $\bar{B} \rightarrow K \ell^{+} \ell^{-}$, Phys. Rev. D 64, 074014 (2001).

[46] W. Altmannshofer, P. Ball, A. Bharucha, A. J. Buras, D. M. Straub, and M. Wick, Symmetries and asymmetries of $B \rightarrow K^{*} \mu^{+} \mu^{-}$decays in the Standard Model and beyond, J. High Energy Phys. 01 (2009) 019.

[47] A. Crivellin, G. D'Ambrosio, and J. Heeck, Addressing the LHC flavor anomalies with horizontal gauge symmetries, Phys. Rev. D 91, 075006 (2015).

[48] D. Das, C. Hati, G. Kumar, and N. Mahajan, Towards a unified explanation of $R_{D^{(*)}}, R_{K}$ and $(g-2)_{\mu}$ anomalies in a left-right model with leptoquarks, Phys. Rev. D 94, 055034 (2016).

[49] B. Bhattacharya, A. Datta, J.-P. Guvin, D. London, and R. Watanabe, Simultaneous explanation of the $R_{K}$ and $R_{D^{(*)}}$ puzzles: A model analysis, J. High Energy Phys. 01 (2017) 015.

[50] D. Choudhury, A. Kundu, R. Mandal, and R. Sinha, Minimal Unified Resolution to $R_{K^{(*)}}$ and $R\left(D^{(*)}\right)$ Anomalies with Lepton Mixing, Phys. Rev. Lett. 119, 151801 (2017).

[51] D. Choudhury, A. Kundu, R. Mandal, and R. Sinha, $R_{K^{(*)}}$ and $R\left(D^{(*)}\right)$ anomalies resolved with lepton mixing, Nucl. Phys. B933, 433 (2018).

[52] S. Bhattacharya, A. Biswas, Z. Calcuttawala, and S. K. Patra, An in-depth analysis of $b \rightarrow c(s)$ semileptonic observables with possible $\mu-\tau$ mixing, arXiv:1902.02796.

[53] Y. Afik, J. Cohen, E. Gozani, E. Kajomovitz, and Y. Rozen, Establishing a search for $b \rightarrow s \ell^{+} \ell^{-}$anomalies at the LHC, J. High Energy Phys. 08 (2018) 056.

[54] B. C. Allanach, T. Corbett, M. J. Dolan, and T. You, Hadron collider sensitivity to fat flavourful $Z^{\prime}$ s for $R_{K^{(*)}}$, J. High Energy Phys. 03 (2019) 137.

[55] A. Cerri et al., Opportunities in flavour physics at the HL-LHC and HE-LHC, arXiv:1812.07638.

[56] A. Greljo and D. Marzocca, High- $p_{T}$ dilepton tails and flavor physics, Eur. Phys. J. C 77, 548 (2017).

[57] A. Arbey, T. Hurth, F. Mahmoudi, and S. Neshatpour, Hadronic and new physics contributions to $b \rightarrow s$ transitions, Phys. Rev. D 98, 095027 (2018). 
[58] K. Cheung, W.-Y. Keung, and P.-Y. Tseng, Lepton-flavorviolating $Z^{\prime}$ using the electron-muon channel at the lhc, Phys. Rev. D 94, 075006 (2016).

[59] Z.-R. Huang, Y. Li, C.-D. Lu, M. A. Paracha, and C. Wang, Footprints of new physics in $b \rightarrow c \tau \nu$ transitions, Phys. Rev. D 98, 095018 (2018).

[60] D. A. Faroughy, A. Greljo, and J. F. Kamenik, Confronting lepton flavor universality violation in B decays with high- $p_{T}$ tau lepton searches at LHC, Phys. Lett. B 764, 126 (2017).

[61] G. Aad et al. (ATLAS Collaboration), Search for a heavy narrow resonance decaying to $e \mu, e \tau$, or $\mu \tau$ with the ATLAS detector in $\sqrt{s}=7 \mathrm{TeV} p p$ collisions at the LHC, Phys. Lett. B 723, 15 (2013).

[62] M. Aaboud et al. (ATLAS Collaboration), Search for new phenomena in different-flavour high-mass dilepton final states in pp collisions at $\sqrt{s}=13 \mathrm{Tev}$ with the ATLAS detector, Eur. Phys. J. C 76, 541 (2016).

[63] M. Aaboud et al. (ATLAS Collaboration), Search for lepton-flavor violation in different-flavor, high-mass final states in $p p$ collisions at $\sqrt{s}=13 \mathrm{TeV}$ with the ATLAS detector, Phys. Rev. D 98, 092008 (2018).

[64] ATLAS Collaboration, CERN Technical Report No. ATLASCONF-2017-027, 2017.

[65] G. Aad et al. (ATLAS Collaboration), Search for leptonflavour-violating $\mathrm{H}$ decays of the Higgs boson with the ATLAS detector, J. High Energy Phys. 11 (2015) 211.

[66] A. M. Sirunyan et al. (CMS Collaboration), Search for a singly produced third-generation scalar leptoquark decaying to a $\tau$ lepton and a bottom quark in proton-proton collisions at $\sqrt{s}=13 \mathrm{TeV}$, J. High Energy Phys. 07 (2018) 115.

[67] M. Tanabashi et al. (Particle Data Group), Review of particle physics, Phys. Rev. D 98, 030001 (2018).

[68] R. Aaij et al. (LHCb Collaboration), Search for the leptonflavour-violating decays $B_{s}^{0} \rightarrow \tau^{ \pm} \mu^{\mp}$ and $B^{0} \rightarrow \tau^{ \pm} \mu^{\mp}$, arXiv:1905.06614.

[69] R. Aaij et al. (LHCb Collaboration), Search for the Decays $B_{s}^{0} \rightarrow \tau^{+} \tau^{-}$and $B^{0} \rightarrow \tau^{+} \tau^{-}$, Phys. Rev. Lett. 118, 251802 (2017).

[70] A. Alloul, N. D. Christensen, C. Degrande, C. Duhr, and B. Fuks, FeynRules 2.0-A complete toolbox for tree-level phenomenology, Comput. Phys. Commun. 185, 2250 (2014).

[71] N. D. Christensen and C. Duhr, FeynRules-Feynman rules made easy, Comput. Phys. Commun. 180, 1614 (2009).

[72] J. Alwall, M. Herquet, F. Maltoni, O. Mattelaer, and T. Stelzer, MadGraph 5: Going beyond, J. High Energy Phys. 06 (2011) 128.

[73] T. Sjostrand, S. Mrenna, and P.Z. Skands, PYTHIA 6.4 physics and manual, J. High Energy Phys. 05 (2006) 026.

[74] R. D. Ball et al., Parton distributions with LHC data, Nucl. Phys. B867, 244 (2013).
[75] B. Fuks, M. Klasen, F. Ledroit, Q. Li, and J. Morel, Precision predictions for $Z^{\prime}$-production at the CERN LHC: QCD matrix elements, parton showers, and joint resummation, Nucl. Phys. B797, 322 (2008).

[76] B. Fuks and R. Ruiz, A comprehensive framework for studying $W^{\prime}$ and $Z^{\prime}$ bosons at hadron colliders with automated jet veto resummation, J. High Energy Phys. 05 (2017) 032 .

[77] I. Dorner and A. Greljo, Leptoquark toolbox for precision collider studies, J. High Energy Phys. 05 (2018) 126.

[78] J. de Favereau, C. Delaere, P. Demin, A. Giammanco, V. Lemaître, A. Mertens, and M. Selvaggi (DELPHES 3 Collaboration), DELPHES 3, A modular framework for fast simulation of a generic collider experiment, J. High Energy Phys. 02 (2014) 057.

[79] M. Cacciari, G. P. Salam, and G. Soyez, FastJet user manual, Eur. Phys. J. C 72, 1896 (2012).

[80] V. Khachatryan et al. (CMS Collaboration), Study of Vector Boson Scattering and Search for New Physics in Events with Two Same-Sign Leptons and Two Jets, Phys. Rev. Lett. 114, 051801 (2015).

[81] ATLAS Collaboration, Standard Model summary plots spring 2019, Technical Report No. ATL-PHYS-PUB2019-010, CERN, Geneva, 2019.

[82] G. Cowan, Two developments in tests for discovery: Use of weighted Monte Carlo events and an improved measure, Proceedings of the Progress on Statistical Issues in Searches, SLAC, 2012.

[83] G. Cowan, K. Cranmer, E. Gross, and O. Vitells, Asymptotic formulae for likelihood-based tests of new physics, Eur. Phys. J. C 71, 1554 (2011).

[84] R. D. Cousins, J. T. Linnemann, and J. Tucker, Evaluation of three methods for calculating statistical significance when incorporating a systematic uncertainty into a test of the background-only hypothesis for a poisson process, Nucl. Instrum. Methods Phys. Res., Sect. A 595, 480 (2008).

[85] S. K. Agarwalla, K. Ghosh, N. Kumar, and A. Patra, Samesign multilepton signatures of an $\mathrm{SU}(2)_{R}$ quintuplet at the LHC, J. High Energy Phys. 01 (2019) 080.

[86] N. Kumar and S. P. Martin, Vectorlike leptons at the large hadron collider, Phys. Rev. D 92, 115018 (2015).

[87] M. Aaboud et al. (ATLAS Collaboration), Search for new phenomena in final states with an energetic jet and large missing transverse momentum in $p p$ collisions at $\sqrt{s}=$ $13 \mathrm{TeV}$ using the ATLAS detector, Phys. Rev. D 94, 032005 (2016).

[88] M. Aaboud et al. (ATLAS Collaboration), Search for dark matter and other new phenomena in events with an energetic jet and large missing transverse momentum using the ATLAS detector, J. High Energy Phys. 01 (2018) 126. 